\title{
Apuntes para una historia de la catalogación internacional en los siglos XIX y XX
}

\author{
Ignacio López Guillamón \\ Universidad de Extremadura (España) \\ Biblioteca Central (Badajoz)
}

\subsection{Resumen}

Se presenta una revisión bibliográfica de la catalogación en los dos últimos siglos. El objetivo es mostrar cómo se ha formando el concepto de catalogación internacional a partir, primero, de la idea de cooperación entre bibliotecas; y, segundo, a partir de la normalización de los procedimientos de catalogar. Se presenta el desarrollo de los códigos de catalogación que mayor influencia han ejercido en este proceso, así como las personas, instituciones y asociaciones profesionales que han marcado las pautas de esa evolución. Si hoy es posible hacer una catalogación más allá de fronteras idiomáticas es porque desde hace más de dos centurias se han ido sumando iniciativas en aras de conseguir un código de catalogación internacional.

Palabras clave: Descripción bibliográfica. Catalogación internacional. Historia. Códigos de catalogación. Normalización bibliográfica.

\subsection{Abstract}

An evolution of cataloguing in the last two centuries is presented. The object is to show how the concept of international cataloguing originated in the idea of cooperation among libraries and the normalization of cataloguing procedure. The cataloguing codes that have exerted more influence in this process have been studied, considering the persons, institutions and professional institutions that have guided this evolution. A cataloguing practice beyond idiomatic frontiers is nowadays possible because for more than two centuries different initiatives have been added in order to obtain an international cataloguing code.

Keywords: Bibliographic description. Bibliographic standardization. Cataloguing. Cataloguing codes. International cataloguing. History,

\section{Introducción}

La teoría y la práctica de la catalogación está despertando un interés creciente en la comunidad de profesionales e investigadores de la Biblioteconomía. Sólo durante el pasado año se celebraron la International Conference Authority Scire. $10: 1$ (en.-jun. 2004) 121-144. 
Control: Definition and International Experiences en Florencia del 10 al 12 de febrero; el International Meeting of Experts on an International Cataloguing Code en Frankfurt del 28 al 30 de julio, auspiciado por la IFLA Cataloguing Section; y el International Seminar on Coopetarive Cataloguing \& Authority Control en Hong-Kong el 15 de noviembre. En la misma línea, los resultados de búsquedas bibliográficas y de recursos documentales en bases de datos, LISA, o en Internet son cuantitativamente abrumadores con relación a los resultados ofrecidos hace sólo cinco años.

El presente trabajo ofrece una muestra de la información encontrada en relación con el concepto de catalogación internacional, que en el presente constituye una expresión ampliamente aceptada y que, a poco que se haga una revisión de la historia de la catalogación, aparece como idea implícita en la aspiración de algunos de los códigos de catalogación del pasado siglo XX, pues constituían reglamentaciones supranacionales. El trabajo se articula en torno a algunas de las codificaciones que más relación guardan con la idea de emprender una catalogación internacional o sobre aquéllas que más incidencia han tenido en el conjunto de este tipo de normas. En algunos casos, los autores personales, las instituciones o eventos que las hayan impulsado serán objeto de un tratamiento breve, que ponga de relieve su aportación en aras de lograr la catalogación internacional. En este proceso, España o lo hispano se muestra, en términos absolutos, en la penumbra de tal empresa. En cualquier caso existe y se hará constar. La presentación formal de los datos se hará siguiendo un esquema cronológico, determinado por las fechas de publicación de los códices catalográficos más relevantes. Para restar linealidad, la narración se modulará a partir de conceptos concatenados —o, simplemente, yuxtapuestos - en virtud de poseer un valor semejante. Se pretende con ello fijar los hitos y algunos matices de un fenómeno que, en el transcurso de doscientos años, se muestra, primero, como una tendencia; segundo, como un esfuerzo frustrado en múltiples ocasiones; y, por último, como una realización próxima.

La idea para conseguir un código de catalogación internacional partió de un grupo de profesionales visionarios que fueron, de hecho, quienes impulsaron las asociaciones profesionales, convertidas en agentes patrocinadores de la modernización de la práctica del trabajo en la bibliotecas. El fin de alcanzar instrumentos catalográficos - de validez, primero, nacional, y, más tarde, universal- para el tratamiento y la gestión de los libros tiene que ver con que éstos, como el conocimiento que contienen, carecen de fronteras. Si embargo, en todos los países, el trabajo del bibliotecario posee un carácter de tradición cultural propia. Este valor localista condiciona todas las actuaciones sobre bibliotecas, que se centran en la resolución de sus problemáticas más próximas y perentorias; y es la causa, también, de que se prolongue hasta los comienzos del siglo XXI la carrera hacia la consecución de un código de catalogación internacional.

Scire. $10: 1$ (en.-jun. 2004) 121-144. 


\section{Los pioneros de las reglamentaciones nacionales}

Frente al desarrollo habido en la elaboración de catálogos, bibliografías e índices bibliográficos a partir del Renacimiento, los indicios del esfuerzo por hacer una descripción bibliográfica unívoca hay que rastrearlos en la publicación de esos catálogos y bibliografías e índices y en la edición de obras de contenidos bibliotecológicos (Norris, 1939, p. 130-141). A partir de la difusión de la impresión de libros y de la generalización de ciertos valores culturales, religiosos y científicos en la Europa del siglo XVI, renace con fuerza el interés por formar bibliotecas y, consecuentemente, las ideas y teorías sobre su organización. Un caso paradigmático fue el de la Librería del Real Monasterio de El Escorial. A su promotor le presentaron distintos informes que son exponentes de una Biblioteconomía incipiente. En ellos no consta referencia alguna sobre los aspectos técnicos de la elaboración de sus catálogos de impresos y manuscritos, preparados por Arias Montano en 1577 (López, 2002a, p. 196-197).

La conceptualización biblioteconómica se irá desarrollando paulatinamente. Hitos en el camino son la obra de Justus Lipsius De bibliothecis sintagma, publicada en Amberes en 1602 (Nelles, 1996, p. 224-242) y deudora de las ideas renacentistas sobre bibliotecas; Advis pour dresser una bibliothèque, de Gabriel Naudé, la obra que más estudios y ediciones ha acaparado desde su publicación en 1627 y que posee ediciones de 1644, 1876, 1963 y 1990 (Revel, 1996, p. 243250); y, finalmente, de los años centrales del siglo XVIII, Dissertationes philologico-bibliographicae, obra de Oliver Legipont, publicada en 1747. De ésta última hay una versión en español, realizada en 1759, que se utilizó para organizar la Biblioteca de san Isidro en Madrid (Miguel, 1987, p. 427-448).

Los progresos de la catalogación bibliográfica se aprecian de forma muy significativa en la evolución trazada por Dorothy M. Norris en los catálogos de la Bodleian Library. Destruidos los fondos bibliográficos de esta biblioteca en 1549, se inicia a partir de 1598 con el apoyo de Thomas Bodley una nueva andadura, en la que se incluye la publicación de varias ediciones del catálogo general de sus fondos en 1605, 1620, 1674, 1738, 1843, etc. A partir del de 1674 se muestra la necesidad de que los asientos bibliográficos estén bien diferenciados. Ello condujo, a su vez, a que se elaborara en 1882 una reglamentación propia, que se reedita varias veces y que perdura hasta 1933 (Norris, 1939, p. 142-157).

Louise N. Malclès indica que la práctica bibliográfica de los primeros tiempos era la consecuencia del interés de los bibliógrafos por los autores y sus obras, y no tanto por el libro en sí mismo. Resalta que los catálogos anteceden a las bibliografías y coexisten con ellas, y que las técnicas de elaboración y utilización eran comunes. Unos y otras se diferencian por la organización de los asientos bibliográficos y comparten el hecho de que los datos gravitan en torno al título. 
Esta misma autora indica cómo los primeros teóricos abordaron en sus obras aspectos como la historia de la imprenta, las reglas catalográficos, la conservación de los libros o las funciones de los bibliotecarios (Malclès, 1989, p. 9, 16).

Al finalizar el siglo XVIII, la nacionalización de bienes llevada a cabo por la Revolución Francesa tendrá consecuencias importantes en la concreción escrita de la primera reglamentación catalográfica, Así, el 15 de diciembre de 1790 la Asamblea Francesa sanciona la Instruction concernant la conservation de manuscrits, chartes, sceaux, livres imprimés...; y en mayo de 1791 ya estaba preparada para su distribución la Instruction pour procéder à la confection du catalogue de chacune des Bibliothèques sur lesquelles les Diréctoires ont dî ou doivent incessamment apposer les scellés, que fue redactada por Jean-Baptiste Massieu (García Ejarque, 1994, p. 89). Pronto - concretamente en 1796-, en cambio, se desechó esta propuesta debida al bibliotecario real Louis François Lefèvre d'Ormesson (Hopkins, 1992, p. 399 y ss.). Hubo otras iniciativas, entre las que deben citarse la de Urbain Dumergue, director del Bureau Bibliographique de Paris en 1793, o la de Henri Gregoire, obispo de Blois, autores de sendos Rapport bibliographique destinados a facilitar la selección y catalogación de los libros y manuscritos nacionalizados (Malclès, 1989, p. 9). Jean-Baptiste Massieu propone la redacción de cédulas a partir del título, autor, lugar de publicación, año, etc. Era un medio rápido para disponer de los asientos bibliográficos que formarían la Bibliographie générale et raisonnée de la France. Se estima que las fichas constituyeron un material de trabajo interno entre los bibliógrafos que las redactaban y que, ordenadas por los apellidos de los autores, se remitirían al Bureau de Bibliographie, responsable de coordinar la edición de la primera bibliografía nacional francesa en sentido moderno (Dussert-Carbone, 1994, p. 26). Esta reglamentación, tan condicionada por las circunstancias, plasma sin más los usos descriptivos de entonces, tal y como reflejan en sus obras arriba citadas Gabriel Naudé u Olivier Legipont.

El siguiente referente constituye ya una reglamentación elaborada. Se debe al italiano Antonio Panizzi, cuya obra Rules for the compiling of the catalogue se publicó en 1841 dentro del General catalogue of the printed book of the British Museum (1841-1881), y constituye la codificación más técnica y completa hasta entonces conocida. El contexto de esta importante obra lo constituyó la remodelación del Department of Printed Books, del British Museum, emprendida en la década de 1830, y las diferencias de criterio que se suscitaron entre Panizzi y el Trustees de esta entidad (Chaplin, 1987, p. 1-16). Entre otras soluciones innovadoras, Antonio Panizzi apostó por fortalecer los aspectos técnicos relativos a la redacción de los asientos bibliográficos bajo los autores personales y corporativos, ya fueran primeras versiones o traducciones; las publicaciones seriadas irían por su título uniforme; y las variaciones del nombre se resolvían escogiendo la manera más antigua por la cual ese autor era conocido (Biagetti, 2001, p. 129-191). 
Pocos años después, en 1853, Charles C. Jewett, en los Estados Unidos, publica su On the construction of catalogues... siguiendo el modelo adoptado en la Library of British Museum y confiriendo un valor esencial a la descripción bibliográfica a partir del título. (Age..., 1975, p. 15). Tras esta reglamentación están los proyectos bibliotecarios de la Smithsonian Institution, que en sus inicios trató de sentar las bases para preparar un catálogo colectivo de las bibliotecas públicas de Estados Unidos y del mundo (London, 1980, p. 253 y ss.). En los años centrales del siglo XIX, el estado de la educación y la ciencia en este país se transforma por la acción gubernativa y las iniciativas privadas. El sistema público de bibliotecas fue concebido e impulsado como apoyo del sistema de escolarización y de promoción social de las oleadas de inmigrantes que llegaban a poblar este país. El proyecto de Jewett obedece a esta política de explotar todos recursos bibliográficos de los Estados Unidos (Biagetti, 2001, p. 203-228).

En el tercer cuarto del siglo XIX, la realidad bibliotecaria de los Estados Unidos era bien diferente de la que llevó al fracaso el ambicioso proyecto en que había participado Charles C. Jewett. Tras la Guerra de Secesión, las bibliotecas públicas y docentes de este país fueron objeto de atención preferente por la administración. Este contexto servirá a Charles A. Cutter para racionalizar la práctica catalográfica. En 1876 publicó Rules for a printed dictionary catalogue, obra que marca la madurez de la práctica de la catalogación hasta entonces y que es un referente para los códigos catalográficos posteriores. Cutter fortalece las entradas bibliográficas y la descripción misma, de forma que hasta la International Conference on Cataloguing Principles de París en 1961 fue el código de mayor influencia sobre el resto de las reglamentaciones (Biagetti, 2001, p.253-281). Para Cutter, las entradas bibliográficas sirven para organizar el catálogo de manera pragmática en virtud de su explotación informativa. Establece de forma categórica la catalogación por las tres entradas tradicionales - autor, título y materias - y refuerza el poder informativo del catálogo clásico como instrumento unitario de acceso a los libros (Solimine, 1995, p. 154-155). Además de Cutter, es necesario reseñar otros autores de codificaciones relevantes, como Andrea Crestadoro o K. Dziatzko (Sharp, 1948, p. 311 y ss.).

En general se constata que el último tercio del siglo XIX fue prolífico en nuevas versiones de reglas catalogáficas en todo Occidente. Así, en los Estados Unidos, la American Library Association publicó en 1883 el Condensed rules for an author and title catalog, revisadas y reeditadas en 1886. En 1884, F. B. Perkins publicó las San Francisco cataloguing for public librairies; y en 1886, Melvil Dewey dió a conocer sus Rules for author and classed catalogs as used in Columbia College Library. El mismo fenómeno se daba en el Reino Unido, dónde, en 1878, Henry Bradshaw redactó en cuarenta y nueve puntos las Rules to be observed in forming the alphabetical catalogue of printed books, de la Cambridge

Scire. $10: 1$ (en.-jun. 2004) 121-144. 
University. De 1883 son las Cataloguing rules de la Library Association; y las Compendious cataloguing rules for the author and titles entries de la Bodleian Library. Las reglamentaciones de ésta y de la Oxford University fueron trazadas por E. W. B. Nicholson en 1882 sobre la base del código de la Library Association de 1883. Ambas constaban de cincuenta y nueve puntos. Diez años más tarde, se publicó el nuevo código de catalogación del British Museum. Por su parte, en Francia se publica en 1878 la Instruction générale relative au service des bibliothèques universitaires. En 1889 Léopold Delisle dio a conocer su Instructions élémentaires et techniques pour la mise et la maintien en ordre des livres d'une bibliotheque, de amplia implantación en las bibliotecas de Francia y de la Bélgica francófona. En España, hay que citar que en 1882 Cándido Bretón y Orozco redactó una Instrucción para formar los índices de impresos de las Bibliotecas administradas por el Cuerpo Facultativo de Archiveros, Bibliotecarios y Anticuarios y que ésta estuvo precedida de la primera edición española de unas reglas catalográficas: la Instrucción para formar los índices de impresos existentes en la Biblioteca Nacional, redactada por don Indalecio Sancha y publicada en 1857 (López, 2001, p. 153-154), aunque se documentan con anterioridad versiones manuscritas (García Ejarque, 1994, p. 89-106). En general, se constata en ellas la influencia que en todos los ámbitos de la administración ejerció el país vecino en gran parte del siglo XIX sobre el nuestro.

Todas estas reglas catalográficas responden bien a las iniciativas administrativas de los países occidentales por desarrollar un sistema de bibliotecas públicas, o bien de algunas instituciones universitarias y de bibliotecas nacionales por disponer de un código propio de catalogación. Tras ellas está el proyecto de reunir en catálogos colectivos nacionales o en bibliografías especializadas el patrimonio bibliográfico, que se multiplicaba en todos los ámbitos del saber bajo el impulso del Positivismo.

En la última década del siglo XIX se asiste a un fenómeno nuevo: la internacionalización de los proyectos catalográficos. En 1895, en Bruselas y en el transcurso de la celebración de la Première Conference Internationale sur la Bibliographie se inició el proyecto de Repertoire Bibliographique Universal, de cuya materialización se ocuparía el Institut International de Bibliographie en las primeras décadas del siglo XX. Éste será el primer ensayo real de catalogación internacional. Como antecedentes limitados de esta iniciativa pueden citarse el Catalogue of Scientific Papers, emprendido por la Royal Society en 1863 y algunos congresos internacionales sobre Matemáticas (1889), Ciencias Naturales, Química Aplicada (1894) o Zoología (1895) (Rayward, 1996, p. 5562). El Repertoire Bibliographique Universal es la iniciativa bibliográfica y de documentación más importante surgida hasta entonces, debida a Paul Otlet y a Henri La Fontaine, y tuvo, como no podía ser de otra manera, un gran impacto en 
la catalogación bibliográfica. El primero recabó la autorización de Melvil Dewey para adaptar la Decimal classification como sistema de clasificación universal de los asientos bibliográficos, y contribuyó a que se adoptaran, finalmente, para su realización las Rules for a dictionary catalog, de Charles A. Cutter. En 1898 la Office International de Bibliographie publicó las Règles pour la rédaction des notices destinées au Répertoire Bibliographique Universel, que fueron preparadas por el Bureau Bibliographique de Paris (Règles..., 1898, port.). En 1907 se reseñan unas reglas en el apartado VIII del Manuel du Répertoire Bibliographique Universel indicando que se destinan a los materiales impresos se los siglos XIX y XX, y que para el resto materiales se recurrirá a las Instructions pour la rédaction d'un inventaire des incunables conservés dans les bibliothèques publiques de France, de Lèopold Delisle, publicadas en 1886 (Manuel..., 1907, p. 98). Con estas iniciativas, el autor belga viene a dar dimensión internacional a unos principios catalográficos que, justo en los inicios del siglo XX, se comienza a pretender que lleguen a ser de uso universal. Pese a que las iniciativas de establecer reglamentaciones de alcance nacional constituirán el rasgo dominante a lo largo del siglo XX, es también cierto que durante esta misma centuria se irá reforzando la iniciativa otletiana de realizar una catalogación internacional (Arnau, 1999, p. 284 y 347-350)

\section{El difícil camino hacia una reglamentación internacional}

\subsection{La primera mitad del siglo $\mathrm{XX}$}

Hasta fines del siglo XIX, la elaboración de los códigos de catalogación había recaído, principalmente, en personas o, en casos muy concretos, en grupos de profesionales. Con el nuevo siglo, las asociaciones de profesionales de alcance nacional y los grupos de trabajo integrados por expertos de distintos países serán los que releven a los pioneros en su estudio y propuesta.

Las asociaciones profesionales norteamericana y británica datan de 1876 y 1877, respectivamente. Ambas surgieron con el afán de resolver colegiadamente las controversias planteadas por los profesionales, impulsar el intercambio de ideas sobre la ciencia biblioteconómica y promover iniciativas tendentes a la cooperación profesional. A partir de 1895, se suma a éstas el Institut International de Bibliographie (IIB), que en su evolución dió lugar a la recientemente desaparecida FID. En 1926 se crea la International Federation of Library Associations (IFLA), que será uno de los principales organismos impulsores del progreso científico y técnico de las bibliotecas y de la Biblioteconomía en todos los países. Dentro del conjunto de asociaciones profesionales relacionadas con la consecución del estado actual del concepto y praxis de la catalogación internacional, es obligado citar al Joint Steering Commitee for Revision of Anglo-American Cataloguing

Scire. $10: 1$ (en.-jun. 2004) 121-144. 
Rules (JSCAACR), surgido en 1966 como creación conjunta de las asociaciones profesionales, respectivas, de los Estados Unidos y del Reino Unido (Reglas..., 1998, p. XI-XVII). Este último y el Committee on Cataloguing, Description \& Acces son los responsables de la actualización períodica de las Anglo-American Cataloguing Rules, las cuales ejercen, por la extensión de su aplicación, por el grado de desarrollo y actualidad de sus normas, el liderazgo entre los códigos de catalogación en el panorama internacional. Sin embargo, a la vista de las iniciativas emprendidas por la IFLA, desde la década de los noventa del siglo XX, en pro de alcanzar una reglamentación catalográfica consensuada a nivel universal, el auténtico código de catalogación internacional está todavía por llegar.

Vistos los nuevos agentes, es normal que el común denominador de las reglamentaciones publicadas hasta los primeros años del siglo XX haya sido conferir al catálogo el valor de instrumento único de acceso y recuperación de la información bibliográfica dentro de parámetros esencialmente nacionales. La normalización que suponía la aplicación de tal o cual normativa era muestra de la cooperación entre las bibliotecas de un país. Esto contribuye a explicar el éxito que tuvo la comercialización de las fichas catalográficas por la Library of Congress o la iniciativa que en tal sentido fue propuesta por el Instituto International de Bibliographie.

Paul Otlet caracteriza el Repertoire Bibliographique Universel (RBU) desde una óptica internacional. El RBU debería estar "organisé sur une base scietifique, coopérative et permanente,.. doit fournir des reseignements sur les publications de tous les temps (universel), de tous les pays (international), relatives à toutes les matières (encyclopédique). C'est un organe de centralisation et de coordination, [...]". Tal sentido tenía la publicación de la filosofía, realizaciones y mecánicas de trabajo del Institut International de Bibliographie a fin de que fuesen ampliamente conocidas (Manuel..., 1907, p. 18-19).

Por su parte, los acuerdos entre los bibliotecarios de los Estados Unidos y del Reino Unido para conseguir una reglamentación catalográfica común responden también a la idea de cooperación (London, 1980, p. 268-269). Ésta cristalizó en las reglas Catalog rules: author and titles entries..., de 1908. El punto de partida fue la Conferencia de Bruselas de 1895; los agentes patrocinadores, la American Library Association y la Library Association británica; y el artífice, un comité formado por profesionales expertos de una y otra asociación. Los trabajos de coordinación fueron complejos y se dilataron hasta el año de su publicación. Finalmente, se hicieron dos versiones, una norteamericana y otra británica. El Comité conjunto siguió de cerca los logros de las bibliotecas prusianas y alemanas y trataron de establecer acuerdos de cooperación con éstas en su aspiración de conseguir un trabajo de amplia repercusión mundial. El texto aprobado mantenía diferencias de criterio en la preparación de las entradas de autor y en el establecimiento de las fuentes de información de las que extraer los elementos de

Scire. $10: 1$ (en.-jun. 2004) 121-144. 
la descripción. Hasta 1978 en que se edita la primera edición indiferenciada para norteamericanos y británicos, las sucesivas ediciones de las Angloamerican cataloguing rules a partir de 1908 - esto es, las de 1941, 1949 o 1968 - son exponentes de la colaboración entre profesionales de distintos países anglosajones por lograr un código de catalogación común y, también, de la lucha por mantener y hacer prevalecer ciertas prácticas de descripción bibliográfica nacionales.

En 1910, tuvo lugar la Conférence International de Bibliographie et de Documentation bajo el patrocinio del Institut International de Bibliographie. Los puntos a tratar en el mismo eran: el estado de la organización bibliográfica en el mundo; las posibilidades de la cooperación en el trabajo científico; la problemática para la elaboración de un código de catalogación internacional, que incluyera la clasificación decimal; y, la organización de una Unión Internacional de Documentación. La amplitud de las pretensiones de la Conferencia son patentes y, por lo mismo, de consecución difícil como se constatará en la realidad (Rayward, 1999, p. 244-246).

Por lo que respecta al código de catalogación internacional, éste se elaboraría a partir del publicado por la American Library y la Library Association en 1908. Sin embargo, tal iniciativa se encontró con el problema de que los bibliotecarios británicos y norteamericanos estaban muy divididos entre aceptar las Catalog rules: author and titles entries... o la cuarta edición de Rules of a dictionary cata$\log$, que Charles A. Cutter había publicado en 1904 y que habían alcanzado una amplia repercusión internacional. Alemanes y franceses pretendieron que la reglamentación de partida fueran sus respectivas codificaciones. La iniciativa, en fin, se frustraría frente a las pretensiones nacionalistas de disponer de reglas propias.

Efectivamente, el primer tercio del siglo XX fue pródigo en la publicación de reglamentaciones catalográficas de alcance nacional. En España se publica en 1902 el primer gran código español de catalogación: las Instrucciones para la redacción de los catálogos en las bibliotecas públicas del Estado, reeditadas en 1926. En 1910 aparecen las reglas para aplicar a los materiales especiales: las Instrucciones para la catalogación de manuscritos, estampas, dibujos originales, fotografías y piezas de música de las bibliotecas públicas. Los Estados Unidos editan en 1905 las Supplementary rules on cataloguing, de la Library of Congress. En Francia, la Association des Bibliothècaires Françaises publica en 1912 las Règles et usages observés dans les principales bibliothèques de Paris...; en 1923 se dota a la Bibliothèque Nationale Française de una nueva reglamentación: Usages suivis dans la rédaction du catalogue général des livres imprimés de la Bibliothèque Nationale. En Italia se publica en 1922 la Regole per la compilazione del catalogo alfabetico. Todos ellos se contraponen a la iniciativa de crear un código de catalogación internacional. 
Es evidente que en esta etapa del siglo XX, la unificación de las reglas catalográficas de aceptación mundial era un empeño ideal (Solimine, 1995, p. 107). La creación de la IFLA en 1926 ayudará en el camino hacia él, impulsando con efectividad la cooperación bibliotecaria internacional a todos los niveles. A instancias suyas se celebró en Roma y Venecia, en 1929, el Primo Congreso Mondiale delle Biblioteche et de Bibliografia. En el transcurso de sus sesiones de trabajo se propuso la creación de la "Bibliotheca bibliographica internacional", a partir de la elaboración de una bibliografía nacional corriente que desembocaría en la "Bibliografía universal". Se trataba de seguir adelante con la propuesta otleliana de llevar a cabo el RBU, ante el desmayo de los apoyos dados en esos años al Institut International de Bibliographie. En las actas de este congreso se recogen las propuestas y debates sobre la unificación de las reglamentaciones catalográficas. Especial significación cobra la ponencia de M. Z. D. Tobolka Projet d'un code international de règles catalographiques, en la que expone las ventajas que supone la catalogación cooperativa a partir de una catalogación uniforme y única, facilitada por la comercialización de las fichas catalográficas conjuntamente con el libro (Atti..., 1931, p. 124-126). Tobolka presentó, además, los principales obstáculos que impedían la consecución de un código de catalogación internacional: la falta de unidad entre las reglamentaciones más extendidas, la variedad de éstas en cada país, el protagonismo de algunos países, o la oposición de las grandes bibliotecas a cambiar el sistema de elaboración de sus catálogos. Se propusieron medidas de unificación en las fichas catalográficas, en el uso de las abreviaturas técnicas, del sistema de transliteración, de las normas para establecer los encabezamientos o de alfabetización de las fichas (Lasso de la Vega, 1950, p. 336-337).

De este primer congreso de la IFLA se derivarán al menos dos avances importantes en el camino hacia un código de catalogación supranacional. En primer lugar, la Association des Bibliothècaires Françaises prepararó unas Règles générales, que supusieron una puesta al día de la edición de 1912 con el afán de que hubieran sido el punto de referencia entre anglosajones y alemanes. Segundo, el Vaticano reactivó la modernización de su biblioteca; y, en este contexto, la Biblioteca Apostolica Vaticana publicó en 1931 un código catalográfico de aspiración universal: las Norme per il catalogo degli stampati, del cual se publicó una segunda edición en 1938. Su dimensión internacional le vendrá de su propia filosofía, pues asume las últimas corrientes en materia de catalogación, imperantes entre italianos, británicos y norteamericanos (Normas..., 1940, p. IX-XI); y de su plan de traducciones: en 1940 al español - con lo cual podría servir para el conjunto de países de habla hispana- y en 1948 al inglés. Razones externas, de tipo político; e internas, de tipo profesional, harán que este código, reputado como antecedente de primer categoría de las Angloamerican cataloguing rules no tuviera el éxito deseado.

Scire. $10: 1$ (en.-jun. 2004) 121-144. 
Hubo un segundo Congrès International des Bibliothèques et de Bibliographie, que se celebró en 1935 entre Madrid y Barcelona. En él se volvió sobre esta iniciativa de elaborar una reglamentación internacional de catalogación y se recomendó insistir hasta alcanzar la "uniformidad internacional de las reglas concernientes al orden alfabético y a la clasificación de títulos en los catálogos...". A tal fin se creó una Subcomisión del Comité Internacional de Bibliotecas, que debería trabajar por la consecución de unas "reglas uniformes de catalogación de libros". Todo lo cual se recoge en Congreso Internacional de Bibliotecas y Bibliografía (Actas..., 1949, p. 190-191). Sin embargo, la cooperación en materia de reglas catalográficas todavía tendría que sortear viejas y nuevas dificultades y muchas décadas.

Como es patente, muchas de las actuaciones de las figuras relevantes en la teoría de la catalogación se ejercían desde las asociaciones profesionales y quedaron difuminadas entre los documentos y las propuestas finales de congresos, que serán en lo sucesivo el medio de trabajo y discusión de las problemáticas bibliotecarias. Estos eventos encauzarán periódicamente todas las innovaciones propuestas por los bibliotecarios con ideas de vanguardia. Otra fórmula de trabajo es a partir de propuestas concretas dadas por un organismo, que son sometidas al análisis y discusión por grupos de expertos para, finalmente, ser avaladas e impulsadas por la propia entidad profesional entre las instancias administrativas de las que dependen las bibliotecas en todas sus categorías.

\subsection{La segunda mitad del siglo $\mathrm{XX}$}

El análisis de los trabajos de Julia Pettee o de Andrew D. Osborn podría constituir el pórtico de este apartado. Sin embargo, se centrará la atención en la obra de Seymour Lubetzky, que se destaca como el principal teórico occidental en materia de catalogación (Lubetzky..., 2001, p. XI-XVIII). En 1946 la dirección del Processing Department de la Library of Congress publicó Studies of descriptive cataloguing . Este informe se conoce como "Report Henkle", en honor de Herman H. Henkle, director del Processing Department, pero en realidad fue preparado por Seymour Lubetzky, quien analiza el estado de la catalogación en los EE.UU. y de los códigos catalográficos norteamericanos; y concluye que los elementos que conforman la descripción bibliográfica exigían una revisión de las fuentes de información de las que éstos se sacaban y que los mismos deberían presentarse conforme a un plan preestablecido (Solimine, 1995, p. 136). En 1953, Lubetzky publica Cataloging rules and principles: a critique of the ALA Rules for entry and a proposed desing for their revision. Esta obra viene a ser una argumentación contra los códigos catalográficos fundados en la recopilación y sistematización de la casuística, como era el caso de las ALA Rules, de 1949, y a favor de sentar unos principios generales que sirvieran para resolver conjuntamente distintos supuestos de descripción bibliográfica (Lubetzky, 1953, p. 14-36).

Scire. $10: 1$ (en.-jun. 2004) 121-144. 
Como pórtico de la International Conference on Cataloging Principles, de 1961, Lubtzky edita en 1960 el Code of cataloging rules..., en el que recoge la esencia de los principios de la catalogación que se discutirán en la referida conferencia, organizada con la intención de convertir las reglamentaciones de la descripción bibliográfica en un instrumento al servicio de una catalogación funcional y práctica que acabara con la yuxtaposición organizada de supuestos prácticos en la que se habían convertido las reglas de catalogación de la época (Garrido, 1996, p. 103). Según la International Conference on Cataloging Principles (International..., 1981, p. 91-92), en sus sesiones se estudiaron la funcionalidad y problemática de los catálogos. Los expertos en catalogación estudiaron la casuística de las entradas de autores personales y corporativos, las obras de varios autores o con distintos nombres, las transliteraciones de autores orientales, entre otras cuestiones, y acordaron en base a principios válidos para distintos casos la determinación de las entradas y la forma que éstas debían tener. Estos acuerdos serían asumidos por todos los códigos catalográficos siguientes. La conocida entre nosotros como Conferencia Internacional sobre los Principios de Catalogación representa una etapa decisiva en la cadena de reuniones internacionales de 1910, 1929, 1935, 1950 y de los años siguientes, cuyo objetivo era que cada país elaborara una bibliografía nacional redactada con unas reglas de catalogación uniformes. La suma de todas ellas haría posible la bibliografía universal, cumpliendo el objetivo del Control Bibliográfico Universal (CBU). Se basa en que cada estado disponga de una agencia bibliográfica nacional, que reúna las publicaciones realizadas en el país, haga su catalogo y dé a conocer al resto de países su bibliografía nacional corriente y retrospectiva. En los países europeos y occidentales este trabajo ha recaído sobre las bibliotecas nacionales (Anderson, 1984, p. 366-401). Se trataba, en definitiva, una nueva versión del RBU, propuesto por P. Otlet muchas décadas antes. La concepción que hay respecto de un código de catalogación internacional sigue siendo la de un instrumento esencial en la cooperación bibliotecaria.

Otro hito fundamental en el itinerario que lleve hasta lograr una práctica común de la catalogación fue el programa MARC. A partir de 1960, se comienza a experimentar en la Library of Congress con la redacción de los asientos bibliográficos sobre un formato que permitiese su interpretación por sistemas automatizados: el MARC. En 1966 ya era un proyecto piloto al que se sumaron las principales bibliotecas norteamericanas y europeas, que recibían en cintas legibles por ordenador la catalogación realizada por la Descriptive Cataloging Division, de la Library of Congress, como experimentación sobre el intercambio de información bibliográfica (García Melero, 1983, p. 106-108). En la actualidad, el MARC es el formato seguido en la automatización efectiva de los recursos informativos y documentales de numerosas bibliotecas de todo el mundo (Delsey,

Scire. $10: 1$ (en.-jun. 2004) 121-144. 
2001, p. 1-3). Sus desarrollos y posibilidades tienen un amplio futuro (Monreal, 2002, p. 131-152), pese a que las innovaciones tecnológicas ofrecen otras alternativas (Ortiz-Repiso, 1999, p. 198-206).

En 1969 tuvo lugar el International Meeting of Cataloguing Experts, en Copenhague, que se inició con la propuesta de Michael Gorman de articular una estructura para la descripción bibliográfica, que vendría determinada por áreas con los elementos bibliográficos de la descripción separados por signos de puntuación con significación propia (Fonseca, 1973, p. 18). Esta reunión fue la cuna de la descripción normalizada internacional de libros (ISBD) y supuso un paso determinante a efectos de codificar la catalogación de manera que fuera una actividad susceptible de ser automatizada. Los desarrollos, actualizaciones y nuevas ISBDs se han sucedido en el último cuarto del siglo XX (López, 2002b, p. 135-141).

Los últimos hechos citados han exigido una nueva elaboración de las reglamentaciones de catalogación de todos los países. Por la influencia que ejercen las Anglo-American cataloging rules (AACR) sobre el resto de las codificaciones catalográficas, éstas centrarán los comentarios siguientes. Más arriba se indicó que en 1978 se publicó la primera edición única como culminación de un proyecto largamente trabajado. Entre las causas inmediatas de este hecho estaban los sucesos que se acaban de exponer. Sin embargo, sólo diez años después fue necesario publicar una segunda edición revisada, las AACR2. El JSCAACR y el Committee on Cataloguing, Description \& Access efectuaron enmiendas en 1993 y 1997 (Reglas..., 1998, XIII); en 2002 se hizo una nueva revisión. En nuestros días se cuestiona su futuro. Así, con el patrocinio de la Library of Congress tuvo lugar en Washington en noviembre de 2000 la Bicentennial Conference on Bibliographic Control for the New Millenium: Confronting the Challenges of Networked Resources and the Web. En este marco se discutieron las perspectivas de futuro de las AACR2 ante las nuevas propuestas de metadatos, el estado de las autoridades bibliográficas o la tecnología web como medio de presentación de los catálogos, entre otros (Bicentennial..., 2000, p. 1 y ss.). El debate sobre las AACR2 es extensivo a todas las reglamentaciones catalográficas, según se resalta en las ponencias de este evento internacional, toda vez que son consideradas el código de referencia de todas ellas; $y$ en las expuestas en las conferencias celebradas durante el 2003, citadas al comienzo del trabajo.

En el trasfondo de todos estos sucesos late un documento esencial, publicado por la IFLA en 1998 y que es el resultado de la reflexión de los profesionales expertos sobre los retos de la tecnología de la información a la catalogación: los Functional Requeriments for Bibliographic Records (FRBR). Este informe es el magma del que ha de salir el código de catalogación internacional. Desde la $56^{\text {th }}$ IFLA Council General Conference (Estocolmo, 1990), la IFLA había apostado por una catalogación más simple y en consonancia con las posibilidades técnicas

Scire. $10: 1$ (en.-jun. 2004) 121-144. 
actuales. En 1991 se constituyó el Grupo de trabajo sobre FRBR, el cual, tras distintas remodelaciones, finaliza su informe en 1997 (Functional..., 1998, p. 511). Los FRBR son la culminación de las múltiples propuestas hechas durante la segunda mitad del siglo XX para que las reglamentaciones catalográficas fueran breves y esencialmente prácticas.

La trayectoria secular de unificar las normas de catalogación, la automatización de los procesos técnicos de descripción bibliográfica y la actualización de todos estos procesos a las realidades tecnológicas presentes son los tres principales impulsos que allanan el camino que conduce hacia un código de catalogación internacional. $Y$ en este punto nos encontramos en el presente, en el que se debate a nivel mundial el fenómeno de la catalogación bibliográfica, que, desde luego, no será como la que hemos visto gestarse y como la que se aplica, todavía hoy, en tantas bibliotecas. Catalogación internacional ya se hace de alguna forma desde el momento que se aplica el MARC, las ISBD o desde que es posible, como sucede de hecho por el protocolo Z39-50, que los registros bibliográficos migren por cualquier sistema informático. La catalogación traspasa fronteras políticas e idiomáticas pero todavía no es elaborada sobre la base de unas reglas únicas. Todos estos logros son consecuencia del esfuerzo dedicado a la cooperación entre bibliotecas en esta materia. El itinerario sobre la evolución de la catalogación bibliográfica permanece abierto en pos de lograr ese esquivo código que permita hacer realidad cien años después el proyecto otleliano, en cuyas palabras: "L'objet principal des travaux de l'Institut International de Bibliographie es la préparation du Répertoire Bibliográphique Universel, inventaire classé, aussi complet et détaillé que possible, des publications dont l'ensemble forme le corps matériel de la littérature et des sciences...." (Manuel..., 1907, p. 18).

La International Conference Authority Control, celebrada en Florencia en febrero de 2003 repasó en sus cinco sesiones los aspectos teóricos y prácticos de una catalogación que ha sido transformada por efecto de la tecnología de la información y que se caracteriza por el valor que han adquirido los procesos automáticos de elaborar un registro bibliográfico sobre unos recursos en constante renovación formal y conceptual. En la era digital, el acceso y recuperación de la información sólo es posible si se encuentran soluciones universales a las autoridades bibliográficas (Guerrini, 2003, p. 1-3). Tal fin persiguen realizaciones como FRANAR, ASAAR (CPF), EAC, LEAF, NACO, SACO, MACS, FAST, etc.

El Cataloguing Code Comparison for the IFLA Meeting of Experts on an International Cataloguing Code ha supuesto un estado de la cuestión de los códigos de catalogación en uso por las agencias bibliográficas nacionales de Occidente, cuyos resultados se presentaron en Berlín en agosto de 2003. Los objetivos eran "to examine cataloguing codes currently in use in Europe to compare their similarities and differences to see if we could get closer together and

Scire. $10: 1$ (en.-jun. 2004) 121-144. 
perhaps develop an International Cataloguing Code" (Tillet, 2003, p. 1-3). Dada la pretensión, hay previstos encuentros de expertos en catalogación de América Central y del Sur para 2004 y para el resto de las grandes regiones de la Tierra hasta el 2007.

\section{España y la catalogación internacional}

En todo el proceso descrito, las referencias a España han sido escasas y de poca relevancia. Realmente no es la catalogación un campo en el que los españoles hayan hecho hasta ahora aportaciones de transcendencia internacional. Con todo, puede interesar a los estudiosos que se reseñen, al menos, algunos hechos, que van más allá de constatar la actualización de las reglas de catalogación españolas conforme al modelo francés, alemán o norteamericano y que, en realidad, son poco conocidos. Más arriba se han hecho referencias a las reglamentaciones españolas publicadas hasta 1910.

La celebración en España del II Congrès International des Bibliothèques et de Bibliographie, celebrado en Madrid y Barcelona entre los días 20 y 30 de mayo de 1935, es un hecho altamente significativo. Pocos años antes, en 1929, tuvo lugar el primero en Roma y Venecia. Ambos fueron patrocinados por la recién creada Federación Internacional de Asociaciones de Bibliotecarios, que, entre su fines, se proponía desarrollar la Biblioteconomía y las bibliotecas a partir de grupos de trabajo específicos y por la convocatoria regular de congresos en torno a un aspecto de la profesional. El tema de reflexión que centró las intervenciones de los congresistas en 1935 fue el préstamo internacional entre bibliotecas. También se trataron colateralmente asuntos como el de las bibliotecas especiales, la formación de los profesionales, la cooperación bibliotecaria o las publicaciones especializadas. Como se indicó más arriba, E. de Grolier propuso..."faire une nouvelle tentative pour parvenir à une uniformité internationale des règles concernant l'ordre alphabétique et la classification des titres dans les catalogues. [...] qu'un système international de catalogue serait un instrument précieux. "... "a recommandé de constituer une Commission du Comité International des Bibliothèques qui s'occupera, selon les directives mentionnées, de la question de règles uniformes de catalogue des livres, [...]" (Actas..., 1949, p. 186). Todavía no se había configurado la expresión "catalogación internacional". En este marco, ante representantes oficiales y numerosos bibliotecarios de todos los países del mundo, pronunció don José Ortega y Gasset el discurso inaugural Misión del bibliotecario, realidad y profecía de la biblioteca y objeto de estudio por tantos profesionales posteriores (Actas..., 1949, p. 79-123). Otra aportación, breve y significativa, fue la realizada por Lasso de la Vega: La formación profesional del bibliotecario, que es todavía una propuesta inteligente y realista de la formación de base y técnica de los bibliotecario que dirigen bibliotecas (Actas..., 1949, p.

Scire. $10: 1$ (en.-jun. 2004) 121-144. 
286-288). A este evento asistieron congresistas de cada provincia. No se ha hecho una investigación sobre lo que supuso la participación masiva de los facultativos españoles en este primer congreso celebrado en España de transcendencia internacional. Ante esta ausencia, sólo cabe indicar que, por las trayectorias de bastantes profesionales, lo que sucedía más allá de nuestras fronteras en relación con la Biblioteconomía interesaba y, en tantos casos, se conocía. Sin embargo, las circunstancias históricas nos alejaban de recibir y participar activamente en la evolución de la profesión bibliotecaria a nivel internacional (Ruiz, 1958 pp. vv.) La propia edición de las actas se demoró hasta 1949 y se hizo "a requerimiento de la Federación Internacional” (Actas..., 1949, p. anteportada).

Tras la Segunda Guerra Mundial toman un nuevo impulso todas las iniciativas de reconstrucción de Europa y el fomento de acciones de alcance supranacional. En este contexto, surge otra oportunidad para el intercambio de ideas y la cooperación internacional en materia de bibliotecas. La IFLA reanudó sus convocatorias anuales. Para 1947 convocó su XIII Reunión Anual del Comité Internacional de Bibliotecarios y Documentalistas, a celebrar en Oslo. Se invitó a España para que enviara una delegación. Sin embargo, hubo una contraorden de la IFLA que indignó a las autoridades españolas hasta el punto de que los responsables de la política nacional de archivos y bibliotecas determinaron que no se participara más en eventos relacionados con esa organización. En 1949, la IFLA patrocinó otra reunión de similar alcance en Basilea y, en virtud de que ésta había de servir para la preparación de la de Washington de 1950, que había de tratar sobre la simplificación y unificación de las reglamentaciones catalográficas, los responsables de la IFLA insistieron ante las autoridades españolas para que nuestro país estuviera representado. Finalmente, se accedió y hubo representación española en la convocatoria de Suiza (Tortajada, 1949, p. 389-394). En cambio, no consta que hubiera participación española en el evento de la capital norteamericana.

En la primavera de 1950 se celebró en España el I Congreso Nacional de Archiveros, Bibliotecarios y Arqueólogos. No era la primera vez que en España los bibliotecarios, archiveros y museólogos exponían los resultados de sus estudios y consideraciones profesionales. Al respecto debe mencionarse que en octubre de 1923 debería haberse celebrado una Asamblea del Cuerpo de Archiveros, Bibliotecarios y Arqueólogos, desautorizada por R.O. de 8 de octubre de ese año, y cuyos resultados se publicaron entre 1923 y 1924 (Comunicaciones..., XXVII; XXVIII). La tónica y el alcance de las problemáticas planteadas en uno y otro evento son fiel reflejo del estado de aislamiento en que se encontraban los profesionales españoles del Cuerpo Facultativo.

Frente a estas realidades, un original trabajo de Francisco Javier Lasso de la Vega pone el contrapunto. Éste versaba sobre el tema de fondo que reunió a los bibliotecarios de los principales países en la capital de Estados Unidos: la unifi- 
cación de las reglas de catalogación. En él se hace eco de los obstáculos que los profesionales de los países europeos y los norteamericanos pusieron al propósito de disponer de un "código internacional de reglas para la catalogación". Lasso de la Vega es el primer profesional español que emplea esta expresión, relativamente usada en lengua francesa (code international de régles catalographiques) y más frecuente de hallar en lengua inglesa (international cataloguing code). Hay, no obstante, un antecedente cubano de 1942 - año de publicación de sus reglas de catalogación - y cuyo título es Reglas internacionales de catalogación... (Lasso de la Vega, 1952, p. 716). Este código se inspira en la edición angloamericana de 1941 y en la traducción española de las reglas vaticanas de 1940. Todavía plantea este trabajo otra innovación realmente pionera en nuestra lengua. A saber, la enumeración de siete principios, que debían caracterizar cualquier reglamentación catalográfica: uniformidad formal, unificación de contenidos, compensación entre encabezamiento principal y secundarios, identificación suficiente de las autoridades bibliográficas, justificación de los datos consignados y extraídos de fuentes externas, compleción - término inventado por él que alude a que la catalogación integrará a todos los elementos bibliográficos con independencia de que consten, o no, en la obra catalogada - , y, finalmente, calificación para orientar al usuario sobre su uso a partir de "una o dos palabras". Todos ellos estaban implícitos en la mayoría de las reglamentaciones de catalogación (Lasso de la Vega, 1950, p. 335-344).

No sólo se daba la espalda a la convocatoria de la IFLA, sino que además se trató de emularla. Las autoridades nacionales de archivos y bibliotecas convocaron a comienzos de 1952 un Congreso Internacional sobre Archivos, Bibliotecas y Propiedad Intelectual (CIABPI) entre los países hispánicos, que habría de tener lugar entre el 20 de octubre y el 3 de noviembre de ese año, conforme al decreto legislativo de 22 de febrero de 1952. Éste fue todo un alarde. Sus actas se publicaron en tres volúmenes y, probablemente, fue el hecho más modesto de los que tuvieron lugar en el mismo. Constó de cuatro secciones: Archivos, Bibliotecas, Propiedad intelectual y Asuntos generales. Los contenidos a tratar pretendían ser una puesta al día en todos los temas profesionales en boga en esos años: instalación y conservación de los fondos documentales, cooperación internacional para la elaboración de instrumentos de descripción de archivos o bibliotecas, creación de un sistema de clasificación de los países hispanos, impulso de la política de difusión del libro, protección del derecho de autor, preparación de un estatuto profesional, etc. (I Congreso..., 1952, p. 103-116). Con relación al tema de este trabajo, hay que significar que el Congreso de 1952 constituyó el principal acontecimiento habido en España desde el II Congreso Internacional de Bibliotecas y Bibliografía, de 1935. Este congreso aspiraba a polarizar la atención internacional en el ámbito de los profesionales de bibliotecas con el mismo énfasis que lo

Scire. $10: 1$ (en.-jun. 2004) 121-144. 
hacía la IFLA o la Unesco. Así, el primer apartado de la Sección de bibliotecas versaba sobre el Estudio de una reglas unificadas de catalogación (impresos, estampas, piezas de música, mapas, microfilm) para todos los países de lengua española y portuguesa. Sobre el mismo se presentaron veintiuna ponencias que trasladaron a España, desde el tamiz de Argentina y Cuba, principalmente, la actualidad sobre la simplificación de las reglas de catalogación y la necesidad de preparar una reglamentación de aceptación internacional. Las intervenciones oficiales del congreso por parte española abogaban por elaborar más bien unas reglas de alcance hispano, aunque no faltaran intervenciones que abogaran por una apertura más internacional. Los estudios de fondo sobre un código de catalogación unificado correspondieron a Emma Linares, de Argentina, que centró su exposición en analizar cómo se trataban las entidades corporativas en las distintas reglamentaciones y en proponer una unificación que aunara las diferencias entonces existentes (Ponencia 24). Lilia Castro de Morales, de la Biblioteca Nacional de Cuba, propone directamente que se adopten las ALA Cataloging rules (1949) y el Sistema Dewey (Ponencia 34). Víctor M. Guerra, de Guayaquil, disertó sobre los beneficios de disponer de un código de catalogación común para los países hispanoamericanos (Ponencia 127).

Por parte de españoles, se destacan las aportaciones de la Delegación de Barcelona, que sugieren que, en tanto no se disponga de esa reglamentación hispanoamericana, se aplique la $2^{\mathrm{a}}$ ed. de las Instrucciones españolas, publicada en 1941 y reimpresa en 1945 (Ponencia 11). Nicolás Fernández-Victorio, Ángela García Rives y María Luisa Poves resaltaron el valor de la unificación de los encabezamientos y la relación y alcance de las reglamentaciones de la ALA y del Vaticano (Ponencia 38). En esta misma línea se expresó Francisco Javier Lasso de la Vega, conocedor de las técnicas biblioteconómicas en uso en los países hispanoamericanos y en Norteamérica. Él propuso que se aceptaran como código de partida el de la Library of Congress o el de la Biblioteca Vaticana, y que se sometieran a estudio los puntos que entraran en colisión con la tradición catalográfica en lengua española (Ponencia 45). Sobre las dificultades de su materialización trató Abelardo Palanca Pons (Ponencia 134).

La mesa redonda celebrada para finalizar con la Sección de Estudio de unas reglas unificadas... propuso la preparación de unas reglas adaptadas a nuestra lengua, y para ello se proponía tomar como modelo las Normas para la catalogación de impresos de la Biblioteca Apostolica Vaticana (1931), traducidas al español en 1940; el código de catalogación de la Biblioteca Nacional de Argentina; o alguno de los modelos más extendidos entre las normas que se aplicaban en las bibliotecas norteamericanas (Ponencia 27).

Ángela García Rives y María Luisa Poves tuvieron un especial protagonismo en este congreso e hicieron más aportaciones sobre catalogación: un listado con la

Scire. 10: 1 (en.-jun. 2004) 121-144. 
denominación de las entidades corporativas españolas (Ponencia 64); un estudio comparado con soluciones prácticas para la unificación de los encabezamientos de autor, personal y corporativos, tomados de las Instrucciones... españolas, y de los códigos de la ALA, Vaticano y de la Biblioteca Nacional de Argentina (Ponencia 48); o ciertas puntualizaciones concretas para la reforma de algunos apartados de las Instrucciones... españolas (Ponencia 68). Luis Chorro Soria expuso los puntos débiles de las Instrucciones... españolas (Ponencia 151). Otros profesionales abordaron la catalogación desde otras ópticas. Así, Domiciano Herrera Magdaleno expuso unas críticas a la catalogación en vigencia en los años cincuenta (Ponencia 29); Felipe Mateu y Llopis hizo una exposición sobre los tipos de catálogos que debía haber en las bibliotecas españolas (Ponencia 72). Sobre las entidades corporativas habló Fidel Perrino Rodríguez (Ponencia 120) y de los títulos de las publicaciones periódicas, José Alberto Alcorta, de Buenos Aires (Ponencia 79). Otra parte de los trabajos se dedicó a actualizar las Instrucciones... españolas de 1910 sobre los materiales especiales. Tal es el caso de las de los incunables (Ponencia 22), estampas (Ponencia 23), manuscritos (Ponencia 43) y obras musicales (Ponencia 50). Efectivamente, este esfuerzo tuvo fruto: en esa misma década y en la siguiente se publicarán nuevas instrucciones actualizadas de todos los materiales .

Los sistemas de clasificación bibliográficos, alfabéticos y sistemáticos también interesaron. Bastantes trabajos versaron sobre el Sistema de Clasificación Decimal Universal, aplicado a las bibliotecas españolas (Ponencia 28); la numismática en la CBD, por Casto María del Rivero (Ponencia 73); o sobre la problemática de la aplicación de ésta en las bibliotecas, por José Bueno (Ponencia 119). Sobre la clasificación alfabética destacaron las aportaciones de Alfredo Consolé, de Buenos Aires, sobre una nueva clasificación enciclopédica (Ponencia 69); y de Nicolás Fernández-Victorio sobre la dificultad de crear un sistema de clasificación bibliográfico para los países hispanoamericanos (Ponencia 78), así como su propuesta concreta (Ponencia 129). Carlos Huidobro y Viñas expuso las bases del sistema de clasificación de las bibliotecas hispanoamericanas (Ponencia 148). Luis Chorro Soria habló de que el sistema de clasificación bibliográfico hispanoamericano debía ser universal (Ponencia 152); etc. Sobre la asignación de materias alfabéticas presentó una aportación Carmen Rovira, de la Biblioteca Nacional de La Habana (Ponencia 85).

Las pretensiones del congreso fueron todas y los resultados prácticos no tan vistosos como los deseos. En cualquier caso, del esfuerzo intelectual que hicieron tantos bibliotecarios españoles y de las ideas de los bibliotecarios y archiveros venidos de América y Filipinas, dónde la influencia de la biblioteconomía anglosajona era una realidad desde hacía varias décadas, se produjeron, al menos, dos consecuencias inmediatas -y básicas - para los profesionales españoles: el

Scire. $10: 1$ (en.-jun. 2004) 121-144. 
destierro para siempre de la pretensión de hacer una Biblioteconomía hispánica; y la apertura sin reservas hacia la cooperación profesional en los foros internacionales. Todavía puede destacarse una tercera consecuencia: la puesta al día de las Instrucciones españolas para materiales especiales, que se publicarán a lo largo de la década de 1950 (López, 2001b, p. 156-158).

Pese a la idea de crear una Comisión Permanente para hacer un seguimiento de los logros propuestos por este Congreso (Ponencia 94), finalizado éste, las rutinas profesionales, la política sobre bibliotecas de la administración y la realidad misma reubicaron los sueños de los profesionales más ilusionados, que, en lo sucesivo, tomarán parte en los encuentros internacionales, especialmente en la International Conference on Cataloguing Principles, celebrada en París en 1961 (Poves, 1961, p. 29-30). La participación de los profesionales españoles en este importante hito en la evolución de la catalogación en materia de encabezamientos conllevó la tercera y última edición de las Instrucciones para la redacción del catálogo alfabético de autores y obras anónimas en las bibliotecas públicas del Estado, publicadas en 1964.

\section{Conclusiones}

Sucesivas generaciones de expertos en catalogación se han ido cuestionando los principios teóricos y prácticos que han conformado las reglas de catalogación hasta el presente, de forma que constituyen una sistematización dinámica y actualizada, de nuevo, sometida a revisión. La situación actual es la culminación de un proceso complejo, y largo, en el que han sido determinantes las actuaciones personales de los principales teóricos de la catalogación, las aportaciones de las asociaciones y organismos profesionales y, también, los avances tecnológicos.

En los inicios los principios de normalización bibliográfica y de unificación reglamentaria actuaron como estímulos para alcanzar la cooperación bibliotecaria en materia de catalogación por razones pragmáticas. Los principios de la catalogación de uso común, precisión y significación fueron los móviles que han llevado más recientemente a las reglamentaciones catalográficas a disponer de una articulación cohesionada, que ha permitido el libre intercambio de los registros bibliográficos. Este proceso revierte en la universalización de la teoría de la catalogación, sometida en el presente, y en el futuro próximo, a contrastar las tradiciones prácticas, bajo el patrocinio de la IFLA y de las principales instituciones bibliotecarias.

Por lo que respecta a nuestro entorno inmediato, la catalogación en España es deudora de las corrientes catalográficas continentales o anglosajonas, de acuerdo con el predominio de una u otra. En los últimos cincuenta años, excepto la empresa, sin antecedentes ni continuación, de formar una tendencia hispana en 1952,

Scire. 10: 1 (en.-jun. 2004) 121-144. 
los profesionales españoles se han sumado a la corriente de internacionalización de la catalogación.

En el momento actual, se está presumiblemente más cerca que nunca de conseguir un código de catalogación internacional, expresión y concepto que se ha ido consolidando lentamente a lo largo del siglo XX. La tecnología de la información es hoy el principal agente impulsor de la actual renovación y del esfuerzo por depurar la lógica funcional de una reglamentación catalográfica, que se pretende sea el código de catalogación internacional.

\section{Referencias}

The age of Jewett: Charles Coffin Jewett and American Librarianship 1841-1868 (1975).

Ed. Michael H. Harris. Littleton: Librairies Undlimited, 1975.

Anderson, Dorothy (1984). Universal Bibliographic Control. // Encyclopedia of library and information science. New York: Marcel Dekker, 1984. 37, sup. 2, p. 366-401.

Anglo-American cataloguing rules (1978). Chicago: American Library Association, 1978.

Arnau, Pilar (1999). Documentación: hitos históricos, precedentes, Dewey. Otlet, FID.

Madrid: Mundarnau, 1999.

Biagetti, Maria Teresa (2001). Teoria e prassi della catalogazione nominale: i contributi di Panizzi, Jewett e Cutter. [Roma]: Bulzoni, 2001.

Bicenntenial Conference on Bibliographic Control for the Millenium: Confronting the Challenges of Networked Resources and the Weh, 15-17 November, 2000 (2000).

Disponible en: 〈http://lcweb.loc.gov/catdir/bibcontrol/conference.htmp. Consulta: 17 de septiembre de 2003.

Chaplin, A. Hugh. GK: 150 years of the General catalogue of printed books in the British Museum. Aldershot: Scolar Press, 1987.

Comunicaciones enviadas para la Asamblea del Cuerpo de Archiveros, Bibliotecarios y Arqueólogos (1923). // Revista de archivos, bibliotecas y museos. XXVII:10-12 (oct.-dic. 1923) 461-667.

Comunicaciones enviadas para la Asamblea del Cuerpo de Archiveros, Bibliotecarios y Arqueólogos (1924). // Revista de archivos, bibliotecas y museos. XXVIII:1-3 (en.mar. 1924) 1-67.

Congreso Ibero-Americano de Archivos, Bibliotecas y Propiedad Intelectual (1º52.Madrid) (1952). I Congreso Ibero-Americano de Archivos, Bibliotecas y Propiedad Intelectual, Madrid, 20 de octubre - 3 de noviembre de 1952. // Revista de archivos, bibliotecas y museos, 4 a época. LVIII, V:I (en.-mar. 1952) 103-116.

Congreso Mondiale delle Biblioteche e di Bibliografia. Atti di Primo Congreso Mondiale delle Biblioteche e di Bibliografia: [...]. 2 v. Roma: Officine dell'Instituto Poligrafico Stato, 1931.

Congreso Internacional de Bibliotecas y Bibliografía (2º ${ }^{\circ}$ 1935. Madrid-Barcelona) (1949). Actas y trabajos del II Congreso Internacional de Bibliotecas y Bibliografía. Madrid: Asociación de Bibliotecarios y Bibliógrafos de España, 1949.

Scire. $10: 1$ (en.-jun. 2004) 121-144. 


\section{Ignacio López Guillamón}

Delsey, Tom (2000). Functionalanalysis of the MARC 21 Bibliographic and holdings formats Disponible en: <http://www.loc.gov./marc/marc-functional-analysis/ frbrspa.html . Consulta: 04 de enero de 2002.

Dussert-Carbone, Isabelle; [et al.] (1994). Le catalogage: méthode et practiques. I Monographies et publicationes en série. Éd. rev. et augm. París: Cercle de la Librairie, 1994.

Fonseca, Isabel (1973). ISBD (International Standard Book Description). // Boletín de la Dirección General de Archivos y Bibliotecas. 20 (1973) 129-130.

Functional Requeriments for Bibliographic Records: final report (1998). IFLA Study Group on the Functional Requeriments for Bibliographic Records. München: K. G. Saur, 1998.

García Ejarque, Luis (1994). Inicios de la catalogación en España. // Boletín de ANABAD. XLIV:1 (mar.-abr. 1994) 89-106.

García Melero, Luis Ángel (1983). La normalización de los formatos de entrada de datos. // Revista española de documentación científica. 6:2 (abr.-jun. 1983) 105-110.

Garrido, María Rosa (1996). Teoría e historia de la catalogación de documentos. Madrid: Síntesis, 1996.

Guerrini,Mawro_Introduzione al convegno [en línea]. http://www.unifi.it/biblioteche/ac/ en/home.htm $>$. Consulta: 14 de marzo de 2003.

Hagler, R. (1977). Changes in cataloging codes: rules for description. // Library trends. $25: 4$ (1977) 609 ss.

Hopkins, Judith (1992). The 1791 French cataloging code and the origins of de card catalogs. // Libraries and culture. $27: 4$ (1992) 395-403.

International Conference on Cataloging Principles (1961. París) (1981). Ed A. H. Chaplin and Dorothy Anderson. 2a ed., reimp. de 1969 de Clive Bingley. Londres: IFLA, International Office for UBC, 1981.

Lasso de la Vega, Javier (1950). Hacia la elaboración de un código internacional de reglas para la catalogación: [...]. // Revista de archivos, bibliotecas y museos, $4^{\mathrm{a}}$ época. LVI, IV:1-3 (en.-sept. 1950) 335-344 .

Lasso de la Vega, Javier (1952). Manual de Biblioteconomía: organización técnica y científica de las bibliotecas. Madrid: Mayfe, 1952.

London, Gertrude (1980). The place and de role of bibliographic description in general and individual catalogues [...] // Libri. 30:4 (1980) 253-284.

López, Ignacio (2001). Las normativas españolas de catalogación. // Boletín de ANABAD. LI:2 (abr.-jun. 2001) 151-162.

López, Ignacio (2002a). Arias Montano como "Librero mayor". // Torre de los Lujanes. 47 (abr. 2002) 195-214.

López, Ignacio (2002b). El itinerario hacia la catalogación internacional. Trab. inédito depositado en la Fac. de Biblioteconomía y Doc. de la Universidad de Extremadura. Badajoz, 2002.

Lubeztky, Seymour (1953). Cataloging rules and principles: a critique of the ALA Rules for entry and a proposed desing for their revision. Washington: Library of Congress, 1953.

Scire. $10: 1$ (en.-jun. 2004) 121-144. 
Lubeztky, Seymour (2001). Writings on the classical art of cataloging. Comp. by Elaine Svenonius, Dorothy McGarry. Englewood: Librairies Unlimited, 2001.

Malcès, Louise-Noëlie. La bibliographie (1989). Paris: Presses Universitaires de France, 1989.

Manuel du Répertorire Bibliographique Universel (1907) [par] Institut International de Bibliographie. Bruxelles: Institut International de Bibliographie, 1907.

Miguel, Aurora (1987). La obra de Oliver Legipont y la Biblioteca de san Isidro, en Madrid. // Homenaje a Justo García Soriano: miscelánea de estudios con motivo de su jubilación. Madrid: ANABAD, 1987, p. 427-448.

Monreal, José (2002). La saga de los MARC. // José Antonio Magans Wals (coord.). Tratado básico de Biblioteconomía. 4a ed. Madrid: Editorial Complutense, 2002. p. 131-152.

Nelles, Paul (1996). Juste Lipse et Alexandrie: les origines antiquaires de l'histoire des bibliothèques. // Les pouvoir des bibliothèques: la mémoire des livres en Occident. París: Albin Michel, 1996, p. 224-242.

Normas para catalogación de impresos (1940). Ciudad del Vaticano: Biblioteca Apostolica Vaticana, 1940.

Norris, Dorothy M. A history of cataloguing and cataloguing methods 1180-1850: with an introductory survey of Ancient Times. London: Grafton, 1939.

Ortiz-Repiso, Virginia (1999). Nuevas perspectivas para la catalogación: metadotos versus MARC. // Revista española de documentación científica. 22:2 (abr.-jun. 1999) 198-219.

Poves, María Luisa (1961). La Conferencia Internacional sobre Principios de Catalogación. // Boletín de la Dirección General de Archivos y Bibliotecas. X:LXII (nov.-dic. 1961) 29-30.

Rayward, W. Boyd (1996). El universo de la información: la obra de Paul Otlet sobre documentación y organización internacional. Madrid: Mundarnau, 1996.

Rayward, W. Boyd (1999). El universo de la información. 2a . ed. Madrid: Mundarnau, 1999.

Reglas de catalogación angloamericanas (1983). San José: Universidad; Washington: Organización de los Estados Americanos, 1983.

Reglas de catalogación angloamericanas (1998). $2^{a}$ ed., rev. Santafé de Bogotá: Rojas Eberhard, 1998.

Règles pour la redaction des notices destinées au Répertoire Bibliographique Universel (1898) [par] Bureau Bibliographique de Paris [et] publié sous la direction de l'Office International de Bibliographie. Bruxelles: Imprimerie Veuve Ferdinand Larcier, 1898.

Revel, Jacques (1996). Entre deux mondes: la bibliothèque de Gabriel Naudé. // Les pouvoir des bibliothèques: la mémoire des livres en Occident. París: Albin Michel, 1996. p. 243-250.

Ruiz, Agustín (1958). Biobibliografía del Cuerpo Facultativo de Archiveros, Bibliotecarios y Arqueólogos 1858-1958. Madrid: Junta Técnica de Archivos, Bibliotecas y Museos, 1958.

Scire. $10: 1$ (en.-jun. 2004) 121-144. 


\section{Ignacio López Guillamón}

Sharp, Henry A. (1948). Cataloguing: a textbook for use in librairies. London: Grafton, 1948.

Solimine, Giovanni (1995). Introduzione allo studio della Biblioteconomia: riflessioni e documenti. Roma: Vecchiarelli, 1995.

Tillet, Barbara B. (2003). IME ICC: Report of the 1 st Meeting. Francfurt, Germany, July 28-30, 2003 [en línea]_Reported by Barbara B Tillet and the Working Group leaders. Disponible en: 〈http://www.ddb.de/news/ifla_conf_index.htmP. [Consulta: 04 de septiembre de 2003].

Tortajada, Amadeo (1949). La Federación Internacional de Bibliotecarios: España ante el Congreso de Basilea de 1949. Revista de archivos, bibliotecas y museos, 4 a época. LV, III:2 (abr.-jun. 1949), 389-403. 